\title{
Precision Fluid Management in Continuous Renal Replacement Therapy
}

\author{
Raghavan Murugan $^{a}$ Eric Hoste ${ }^{d, e}$ Ravindra L. Mehta ${ }^{c}$ Sara Samoni ${ }^{f}$ \\ Xiaoqiang Ding $^{\text {h }}$ Mitchell H. Rosner $^{\text {b John A. Kellum }}{ }^{\text {a }}$ Claudio Ronco $^{g}$ \\ on behalf of the Acute Disease Quality Initiative (ADQI) Consensus Group
}

\begin{abstract}
${ }^{a}$ Center for Critical Care Nephrology, CRISMA, Department of Critical Care Medicine, University of Pittsburgh School of Medicine, Pittsburgh, Pa., ${ }^{b}$ Division of Nephrolgy, University of Virginia Health System, Charlottesville, Va.,

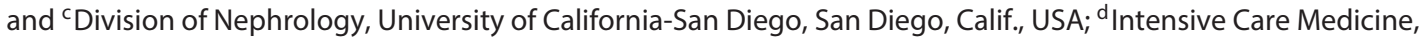
University Hospital Ghent, University of Ghent, Ghent, and ' Research Foundation-Flanders, Brussels, Belgium; ${ }^{\mathrm{f}}$ Institute of Life Sciences, St. Anna School of Advanced Studies, Pisa, and ${ }^{9}$ Department of Nephrology, Dialysis and Transplantation, International Renal Research Institute of Vicenza, San Bortolo Hospital, Vicenza, Italy; ${ }^{\mathrm{h}}$ Department of Nephrology, Shanghai Institute of Kidney and Dialysis, Shanghai Key Laboratory of Kidney and Blood Purification, Shanghai Quality Control Center for Hemodialysis, Zhongshan Hospital, Fudan University, Shanghai, China
\end{abstract}

\section{Key Words}

Fluid - Management - Continuous renal replacement therapy · Precision medicine $\cdot$ Hemodialysis

\section{Abstract}

Fluid management during continuous renal replacement therapy (CRRT) in critically ill patients is a dynamic process that encompasses 3 inter-related goals: maintenance of the

Faculty of the 17th ADQI International Consensus Conference: Bagshaw S.M., Critical Care Medicine, Faculty of Medicine and Dentistry, University of Alberta, Edmonton, Canada; Chakravarthi R.M., Department of Nephrology, STAR Hospitals, Hyderabad, India; De Rosa S., International Renal Research Institute of Vicenza (IRRIV), San Bortolo Hospital, Vicenza, Italy; Floris M., Renal Unit, Ospedale 'G. Brotzu', Cagliari, Italy; Hoste E.A.J., Ghent University Hospital, Ghent University, Ghent, Belgium; Research Foundation Flanders, Brussels, Belgium; Joannidis M., Division of Intensive Care and Emergency Medicine, Department of Internal Medicine, Medical University Innsbruck, Innsbruck, Austria; Mehta R.L., University of California San Diego, San Diego California; Murugan R., Department of Critical Care Medicine, Center for Critical Care Nephrology, University of Pittsburgh School of Medicine, Pittsburgh, USA; Neri M., International Renal Research Institute of Vicenza (IRRIV), San Bortolo Hospital, Vicenza, Italy; Pani patency of the CRRT circuit, maintenance of plasma electrolyte and acid-base homeostasis and regulation of patient fluid balance. In this article, we report the consensus recommendations of the 2016 Acute Disease Quality Initiative XVII conference on 'Precision Fluid Management in CRRT'. We discuss the principles of fluid management, describe various prescription methods to achieve circuit integrity and introduce the concept of integrated fluid balance for tailoring fluid balance to the needs of the individual patient. We sug-

A., Renal Unit, Ospedale 'G. Brotzu', Cagliari, Italy; Osterman M., Guy's and St. Thomas' Hospital, London, UK; Ricci Z., Department of Pediatric Cardiology and Cardiac Surgery, Ospedale Pediatrico Bambino Gesù IRCCS, Roma, Italy; Rosner M., Department of Medicine, University of Virginia, Charlottesville, USA; Samoni S., Institute of Life Sciences, Sant'Anna School of Advanced Studies, Pisa, Italy; Tolwani A.J., Dept of Nephrology, University of Alabama at Birmingham, USA; Xiaoqiang Ding, Department of Nephrology, Shanghai Institute of Kidney and Dialysis, Zhongshan Hospital, Fudan University, Shanghai, China; Wuhua Jiang, Department of Nephrology, Shanghai Institute of Kidney and Dialysis, Zhongshan Hospital, Fudan University, Shanghai, China.

Report from the 17th Acute Disease Quality Initiative (ADQI) Consensus Conference. Asiago, Italy, June 10-13, 2016.

\section{KARGER}

E-Mail karger@karger.com

www.karger.com/bpu (c) 2016 S. Karger AG, Basel

0253-5068/16/0423-0266\$39.50/0
Mitchell H. Rosner, MD

Division of Nephrology

University of Virginia Health System

Box 800133, Charlottesville, VA 22911 (USA)

E-Mail mhr9r@virginia.edu 
gest that these recommendations could serve to develop the best clinical practice and standards of care for fluid management in patients undergoing CRRT. Finally, we identify and highlight areas of uncertainty in fluid management and set an agenda for future research.

(c) 2016 S. Karger AG, Basel

\section{Introduction}

Fluid management is an integral component for managing critically ill patients to correct abnormalities in plasma composition and maintain fluid balance. Several recent studies have established the need for improved strategies for initial fluid resuscitation and for optimizing fluid administration to prevent fluid overload $[1,2]$. The inherent characteristics of critical illness with multi-organ failure presents a unique challenge for fluid management as leaky capillary beds, hemodynamic compromise and large volumes of fluid required to treat the underlying condition result in fluid accumulation that is often difficult to correct in the absence of renal support. Continuous renal replacement therapy (CRRT) techniques provide a unique set of tools for fluid management that can be customized for individual patients.

Fluids are commonly administered during CRRT to maintain the circuit integrity and patency, and enable the operational characteristics for each CRRT modality. The solute content and site of administration of fluids is varied to achieve specific parameters of electrolyte and acidbase balance required for organ support. The volume of fluid removed and infused can be manipulated to provide precise control for fluid balance. This is critical since, at various times, patients may require net fluid removal despite receiving large volumes of obligate fluids for their care (such as antibiotics, nutrition, vasopressors and others). At other times, patients may require net fluid increase to maintain hemodynamic goals. Unlike other forms of renal replacement therapy (RRT) each of these processes can be adjusted independently in CRRT thereby providing enormous flexibility and control for personalized renal support. Despite these inherent capabilities for CRRT, there is wide variation in how these techniques are used in practice. Differences in operational characteristics of CRRT modalities (slow continuous ultrafiltration (SCUF), continuousvenovenous hemofiltration $(\mathrm{CVVH})$, continuous arteriovenous hemodialysis and continuous venovenous hemodiafiltration (CVVHDF)) and the availability of different machines with different user interfaces may contribute to these differences and potentially influ- ence outcomes. There are currently no benchmarks for fluid management with CRRT. Specific standards for fluid management were proposed in the first Acute Disease Quality Initiative (ADQI) conference on CRRT [3], and specific situations were addressed in subsequent conferences [4]; however, there are limited descriptions of best practices for fluid management with these techniques [5].

In 2016, a group of experts in CRRT met at the ADQI XVII consensus conference on 'precision CRRT' and to recommend standard of care/best practices. In this report, we summarize the consensus recommendations on the topic of fluid management with CRRT based on the existing clinical evidence. We also discuss the gaps in our knowledge and identify future research questions to improve fluid management with CRRT in the critically ill patient.

\section{Methods}

The ADQI process has been described previously and complete ADQI methodology description is available at www.adqi.org. Briefly, we convened a 3 days consensus conference in Asiago, Italy, in June 2016. We invited experts in nephrology and critical care medicine from both adult medicine and pediatrics. Principals of current practice were debated in light of existing evidence. Areas of broad consensus were described and a research agenda was developed using a modified Delphi method. Prior to the conference we identified fundamental questions for which consensus would be sought. During the pre-conference phase, each workgroup performed comprehensive literature searches to summarize existing knowledge and to identify gaps that could be addressed by future research. Core questions/concepts were crafted and these were presented to the entire ADQI consensus group during the conference; a series of breakout sessions and plenary presentations allowed debate, discussion and refinement of these concepts. For the timing and patient selection workgroup, literature searches were conducted using the following terms: 'renal replacement therapy' together with either 'acute kidney injury', 'acute renal failure' or 'continuous venovenous hemodialysis'/'hemofiltration' in MEDLINE using PubMed as the search engine. The 4 core questions ultimately addressed by the workgroup are shown in table 1 .

\section{Results}

What Is the Purpose of Various Fluids Used in CRRT? Conceptually, fluids used during CRRT can be classified into 3 inter-related domains (fig. 1). First, fluids are administered to maintain CRRT circuit integrity and patency. Second, the fluid composition can be varied to achieve specific target levels for plasma electrolytes and acid-base balance. Finally, the amount of fluid infused or removed can be adjusted to regulate volume status. Many different types of fluids are used and the site of delivery is 
Table 1. Questions identified by the consensus group

(1) What is the purpose of various fluids used in CRRT?

(2) Circuit integrity

(a) How can fluids be utilized to maximize circuit integrity?

(3) Plasma composition

(a) How can CRRT be used to achieve plasma homeostasis in patients with derangements in plasma composition?

(b) What are the various dialysate and replacement compositions available and how should they be utilized?

(c) How does the use of citrate change the use of dialysate and replacement solutions?

(4) Fluid balance and regulation

(a) What are the principles for fluid management with CRRT?

(b) What are the physiological targets and goals for fluid management with CRRT?

(c) How can fluid removal and balance be achieved?

(5) Precision CRRT

(a) How can the different domains of fluid management be integrated to achieve the goals of care?

(6) Stepwise methodology for prescription of fluid regulation

(a) What are the key components of a CRRT prescription to achieve goals of plasma composition and fluid balance?

(7) Monitoring

(8) Research questions

(a) When is the right time to initiate CRRT for fluid/volume management?

(b) What is best practice to achieve desired fluid balance with CRRT?

(c) What are key technological issues?

Fig. 1. Conceptual framework of fluid management during CRRT. UF heparin = Unfractionated heparin. Reprinted with permission from www.ADQI.org.

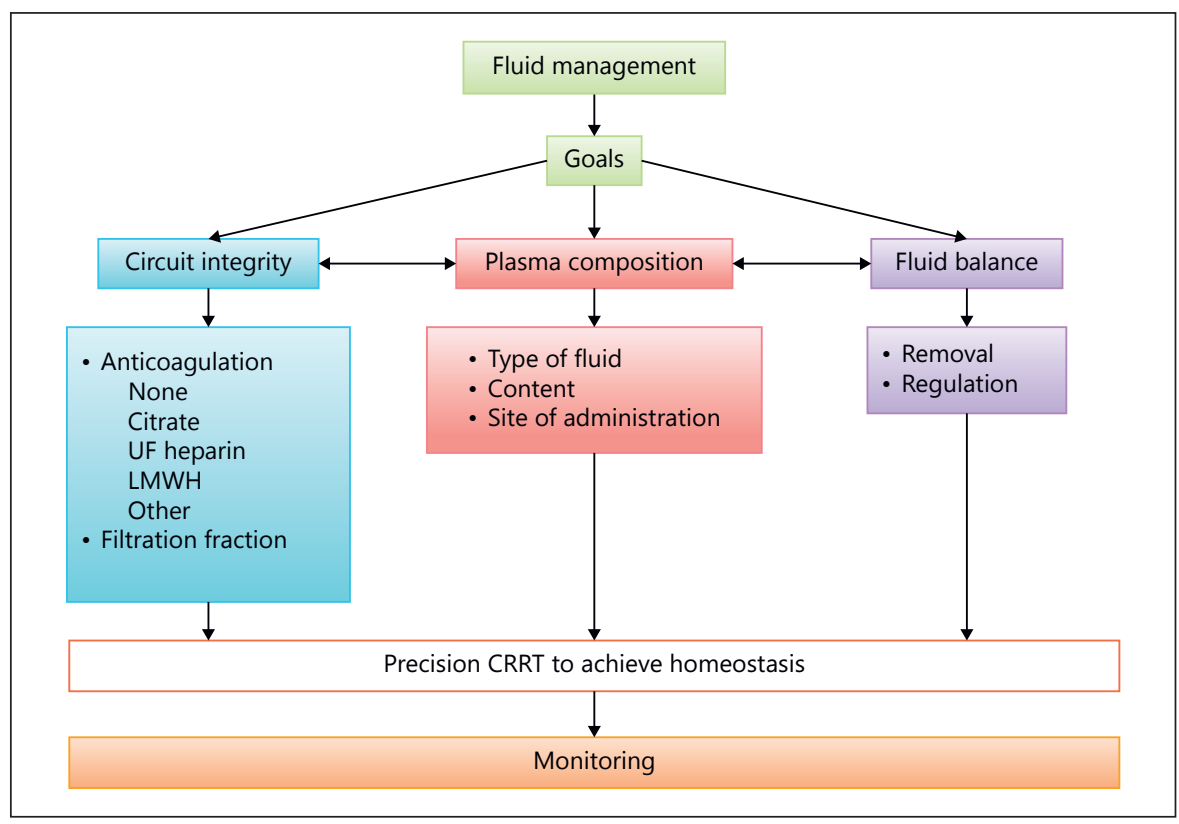

varied depending on the modality of CRRT and the needs of the patient.

In CVVH, fluids are used to replenish plasma volume and electrolytes that are lost during ultrafiltration (UF) and convective removal of solutes ('replacement fluids').
In continuous venovenous hemodialysis (CVVHD), dialysate solutions are used to achieve diffusive clearance. In CVVHDF, a combination of both replacement fluid and dialysate solutions (convective and diffusive clearance) is used to achieve desired results. 
Fluids are administered at various sites in the CRRT circuit and also intravenously (online suppl. fig. S1; for all online suppl. material, see www.karger.com/doi/10.1159/ 000448528). Replacement fluids are administered either pre-filter (pre-dilution), post-filter (post-dilution) or intravenously. Dialysate solutions are typically administered through the dialysis port in the filter. It is important to recognize that these various fluids are interdependent and influence one another, and thus, fluid prescription strategies for delivering precision CRRT need to take into account the type, volume, composition and site of administration of fluids used in all of the 3 domains. This will be discussed in more detail.

\section{Circuit Integrity}

\section{How Can Fluids Be Utilized to Maximize Circuit \\ Integrity?}

Maintaining circuit patency (avoidance of system clotting) is paramount for achieving various intended treatment goals with CRRT. Online supplement figure S1 shows a typical CRRT circuit and its nomenclature. Fluids to maintain circuit patency are typically administered pre-filter and can include saline flushes, replacement fluid (CVVH or CVVHDF) or act as a vehicle for anticoagulants. Anticoagulants such as heparin or citrate are infused upstream of the hemofilter either in a dilute or concentrated form. Citrate (which is converted to bicarbonate in the liver) also acts as a buffer and can be useful in patients with metabolic acidosis $[6,7]$.

Fluids administered before the filter (pre-dilution) are utilized to prevent or reduce filter clotting independent of the anticoagulation used, by lowering the blood viscosity through a decrease in the hematocrit (Hct) and reducing the filtration fraction (FF). FF is expressed as follows:

$$
\mathrm{FF}=\frac{\mathrm{UF} \text { rate }(\mathrm{ml} / \mathrm{min})}{\text { plasma flow rate }\left(\mathrm{Q}_{\mathrm{P}}\right)(\mathrm{ml} / \mathrm{min})}
$$

where, $\mathrm{Q}_{\mathrm{P}}=\mathrm{Q}_{\mathrm{B}}(1-\mathrm{Hct}) ; \mathrm{Q}_{\mathrm{B}}=$ blood flow rate.

An increase in UF rate and Hct or a decrease in plasma flow rate will increase FF and thus increase the risk of filter clotting. A high FF $(>0.25)$ is typically associated with increased risk of poor filter performance and clotting due to hemoconcentration-related effects [8].

Fluids are usually administered pre-filter prior to the pumping of the blood (pre-pump), as post-pump administration or both, to maintain $\mathrm{FF}<0.25$. One major
Table 2. FF at various blood $(\mathrm{Qb})$ and dialysate flow rates as well as varying UF rates

\begin{tabular}{lccll}
\hline Hct & Qb & QP & UF & FF \\
\hline 0.25 & 150 & 112.5 & 1,000 & 0.15 \\
0.35 & 150 & 97.5 & 1,000 & 0.17 \\
0.4 & 150 & 90 & 1,000 & 0.19 \\
0.25 & 200 & 150 & 2,000 & 0.22 \\
0.35 & 200 & 130 & 2,000 & 0.26 \\
0.4 & 200 & 120 & 2,000 & 0.28 \\
0.25 & 150 & 112.5 & 2,000 & 0.30 \\
0.35 & 150 & 97.5 & 2,000 & 0.34 \\
0.4 & 150 & 90 & 2,000 & 0.37 \\
0.25 & 200 & 150 & 1,000 & 0.11 \\
0.35 & 200 & 130 & 1,000 & 0.13 \\
0.4 & 200 & 120 & 1,000 & 0.14 \\
0.25 & 100 & 75 & 1,000 & 0.22 \\
0.35 & 100 & 65 & 1,000 & 0.26 \\
0.4 & 100 & 60 & 1,000 & 0.28 \\
0.25 & 100 & 75 & 2,000 & 0.44 \\
0.35 & 100 & 65 & 2,000 & 0.51 \\
0.4 & 100 & 60 & 2,000 & 0.56 \\
\hline
\end{tabular}

Table 3. Plasma flow and FF for different $\mathrm{Q}_{\mathrm{B}} \mathrm{s}$, UF rates and Hct

\begin{tabular}{lcclll}
\hline \multirow{2}{*}{ Hct, \% } & \multicolumn{2}{c}{$\mathrm{Q}_{\mathrm{B}}=150 \mathrm{ml} / \mathrm{min}$} & & \multicolumn{2}{c}{$\mathrm{Q}_{\mathrm{B}}=200 \mathrm{ml} / \mathrm{min}$} \\
\cline { 2 - 3 } \cline { 5 - 6 } & $\mathrm{Q}_{\mathrm{P}}$ & $\mathrm{FF}$ & & $\mathrm{Q}_{\mathrm{P}}$ & $\mathrm{FF}$ \\
\hline $\mathrm{UF}=1,000 \mathrm{ml} / \mathrm{h}$ & & & & \\
$\mathrm{Hct}=25$ & 112.5 & 0.15 & & 150 & 0.11 \\
$\mathrm{Hct}=35$ & 97.5 & 0.17 & & 130 & 0.13 \\
$\mathrm{Hct}=40$ & 90 & 0.19 & & 120 & 0.14 \\
$\mathrm{UF}=2,000 \mathrm{ml} / \mathrm{h}$ & & & & \\
$\mathrm{Hct}=25$ & 112.5 & 0.30 & & 150 & 0.22 \\
$\mathrm{Hct}=35$ & 97.5 & 0.34 & & 130 & 0.26 \\
$\mathrm{Hct}=40$ & 90 & 0.37 & & 120 & 0.28 \\
\hline
\end{tabular}

limitation of post-dilution CVVH is that it does not influence (decrease) FF, thereby increasing the risk of filter clotting due to hemoconcentration. On the other hand, pre-filter dilution will influence the solute dose delivered as the blood is diluted going into the filter [9]. It should be recognized that current machines display the FF based on a computation of the UF/ $\mathrm{Q}_{\mathrm{B}}$ and consequently underestimate the FF as they do not adjust for the plasma volume.

As shown in tables 2 and 3 (as well as with an online calculator found at www.adqi.net), FF is always lower with lower Hcts and higher $\mathrm{Q}_{\mathrm{B}} \mathrm{s}$ and thus the benefit of 
Table 4. Advantages and disadvantages of pre- and post-filter substitution

Pre-filter

Post-filter

Advantages

UF rate is not limited by $\mathrm{Q}_{\mathrm{B}}$

Enhanced elimination of urea from RBC's

Filter life is increased as the Hct throughout the filter remains low

Filter life is increased which may increase filter lifespan and solute clearance, even though hourly solute clearance is decreased

Disadvantages

Solute concentrations are decreased and thus

clearance is decreased

Clearance of solutes is directly related to UF rate

A higher solute clearance rate is produced

Delivery of specified solutes and concentrations directly to the solution

$\mathrm{RBC}=$ Red blood cell. Adapted from Huang et al. [32].

pre-filter fluids is largely due to the dilution of the blood and Hct. Studies have demonstrated increased circuit life with pre- versus post-filter fluid use, and in one study, this difference was as great as a circuit life of 45.7 versus $16.1 \mathrm{~h}$ with pre- versus post-filter fluids, respectively [10-12]. Table 4 summarizes the advantages and disadvantages of pre- and post-filter fluid replacement.

Statement 1: Fluids administered for circuit patency and anticoagulation need to be included in determining net fluid balance. Fluids containing alkali (e.g., citrate) will also contribute to the buffer source and may require adjustment of other fluid therapy to avoid derangements in acid-base homeostasis (Grade B).

Statement 2: Pre-dilution fluid administration increases filter life and maintains filter patency by reducing FF (Grade A).

\section{Plasma Composition}

\section{How Can CRRT Be Used to Achieve Plasma}

Homeostasis in Patients with Derangements in Plasma Composition?

The entire spectrum of commercially available dialysate and replacement solutions can be used to selectively modulate plasma electrolyte composition and acidbase balance. Furthermore, the content of commercially available solutions can also be customized by an on-site pharmacy and tailored to individual needs of the patient. For instance, additional sodium could be added to a custom made dialysate to achieve a desired serum sodium concentration (e.g., $150-155 \mathrm{mEq} / \mathrm{l}$ ) to maintain UF rate is limited by Qb. You cannot order too much UF
because the end-filter Hct will be too high
Because UF rate is limited by FF you may not reach optimal
dose
Filter life may be decreased by high end-filter Hct high serum osmolarity in patients with cerebral edema [13]. Furthermore, various techniques of CRRT allow flexibility of correction of plasma derangements by varying the site of delivery of replacement fluids. For instance, a replacement fluid with a desired electrolyte concentration can be infused during CVVH directly into the patient.

What Are the Various Dialysate and Replacement Compositions Available and How Should They Be Utilized?

Various quality-controlled commercial dialysate and replacement fluids are available for purchase (usually as 5 liter bags; online suppl. table S1). The dialysate and replacement fluids differ in concentrations of potassium, magnesium, calcium, bicarbonate and dextrose. These variable concentrations allow tailoring of dialysate and replacement fluids to meet the needs of the individual patient. For instance, a patient with serum potassium concentration of $6.8 \mathrm{mEq} / \mathrm{l}$ requiring high-dose pressors may benefit from CVVHD with a dialysate potassium concentration of $0 \mathrm{mEq} / \mathrm{l}$, and this can be modified further depending upon the patient's need and serum electrolyte concentrations. As an example of how fluids used for one purpose may influence the selection of fluids elsewhere in the CRRT circuit, a patient receiving CRRT with regional citrate anticoagulation may require a dialysate with no calcium to maximize the anticoagulant effect of the citrate. Additionally, the amount of bicarbonate needed in the replacement or dialysate solutions may need to be adjusted as citrate metabolism will provide a source of bicarbonate. 
In some instances, unique electrolyte problems may require the use of more custom dialysate or replacement compositions that are not available 'off the shelf. For instance, some patients with severe hyponatremia may require lower dialysate sodium levels at the initiation of therapy to prevent over-rapid correction of the serum sodium [14]. In other circumstances, custom bicarbonate levels may be required or phosphate may need to be added to the dialysate or replacement fluids to correct significant metabolic acidosis or hypophosphatemia. These custom dialysate solutions are typically compounded in a hospital pharmacy and are expensive to produce with a risk for errors [15]. Thus, in centers where custom dialysate solutions are produced, strict quality control measures must be in place to avoid errors in the composition of the dialysate and to ensure sterility.

Patients receiving CRRT in the CVVH or CVVHDF modes require some proportion (usually the majority) of the ultrafiltrate to be replaced with fluids. This is to ensure that the patient does not become volume depleted as well as develop hemoconcentration by continuous UF. As discussed above, the site of delivery either before the dialysis membrane (pre-filter or pre-dilution) or after the dialysis membrane (post-filter or post-dilution) should be based on the desired effects (table 4).

Statement 3: Commercially available dialysate and replacement solutions allow flexibility in achieving various goals of plasma electrolyte compositions to address the majority of clinical circumstances (Grade E).

Statement 4: While we recommend that custommade solutions should be avoided, certain situations may arise that require production of custom dialysate or replacement solutions. In these cases, experienced pharmacists should be available to compound these solutions and strict quality control measures should be in place to ensure that errors do not occur (Grade E).

Statement 5: The choice of dialysate and replacement solution with specific compositions should be based upon the goals for serum electrolyte and acid-base parameters as well as the choice of anticoagulation. The use of dialysate and replacement solutions must be individualized and reassessed at frequent intervals (such as every 6-12 h) depending upon the clinical condition of the patient to ensure that they are appropriate (Grade E).

Statement 6: Replacement solutions should be sterile and dialysate solutions should ideally be sterile or, at a minimum, be ultra-pure (Grade A).

Statement 7: Administration of replacement solutions can be either pre- or post-filter within the dialysis circuit or outside of the circuit (intravenously). Pre-filter replacement of solutions is useful to minimize filter clotting by reducing the FF but influences the dose by decreasing solute concentrations. Post-filter replacement allows direct delivery of solute to the circulation (Grade A).

\section{How Does the Use of Citrate Change the Use of}

Dialysate and Replacement Solutions?

During citrate anticoagulation, sodium citrate is infused into the inflow ('arterial') limb of the extracorporeal circuit, chelating calcium and thereby inhibiting clotting [16]. Intravenous calcium must therefore be infused systemically to maintain a normal ionized serum calcium concentration. Since the citrate provides an alkali load, buffers (e.g., bicarbonate, lactate) may need to be reduced in concentration or deleted from the dialysate and replacement fluids $[6,7]$. The dialysate and replacement fluids may need to be calcium-free to prevent reversal of the citrate effect in the extracorporeal circuit. If a hypertonic citrate solution is utilized, the sodium concentration in the dialysate and/or replacement fluids will need to be reduced to prevent the development of hypernatremia.

Statement 8: During citrate anticoagulation, frequent measurement of serum ionized calcium (post-filter and peripherally) should be done to appropriately titrate the dose of citrate and calcium replacement. Furthermore, systemic acid-base balance should be closely monitored for the development of metabolic alkalosis or citrate accumulation (Grade E).

\section{Fluid Balance and Regulation}

Volume overload (defined as a positive weight gain or a positive value of the total input - total output/initial body weight) is commonly encountered in critically ill patients and is associated with poor outcomes including mortality, length of mechanical ventilation and hospital stay $[17,18]$. The Vasopressin and Septic Shock Trial noted that fluid accumulation values were staggering and ranged from 8 to 30 liters over the first $12 \mathrm{~h}$ of ICU care [1]. Since, fluid overload states are more commonly associated with oliguria and AKI where fluid intake cannot be offset with increases in urine output, mechanical UF utilizing CRRT becomes a viable option to avoid fluid overload and its consequences [19]. This is especially true in those patients who are hemodynamically unstable and may not be able to tolerate large volumes of fluid removal (2-4 liters or more) during a shorter hemodialysis ses$\operatorname{sion}(3-4 \mathrm{~h})$. 


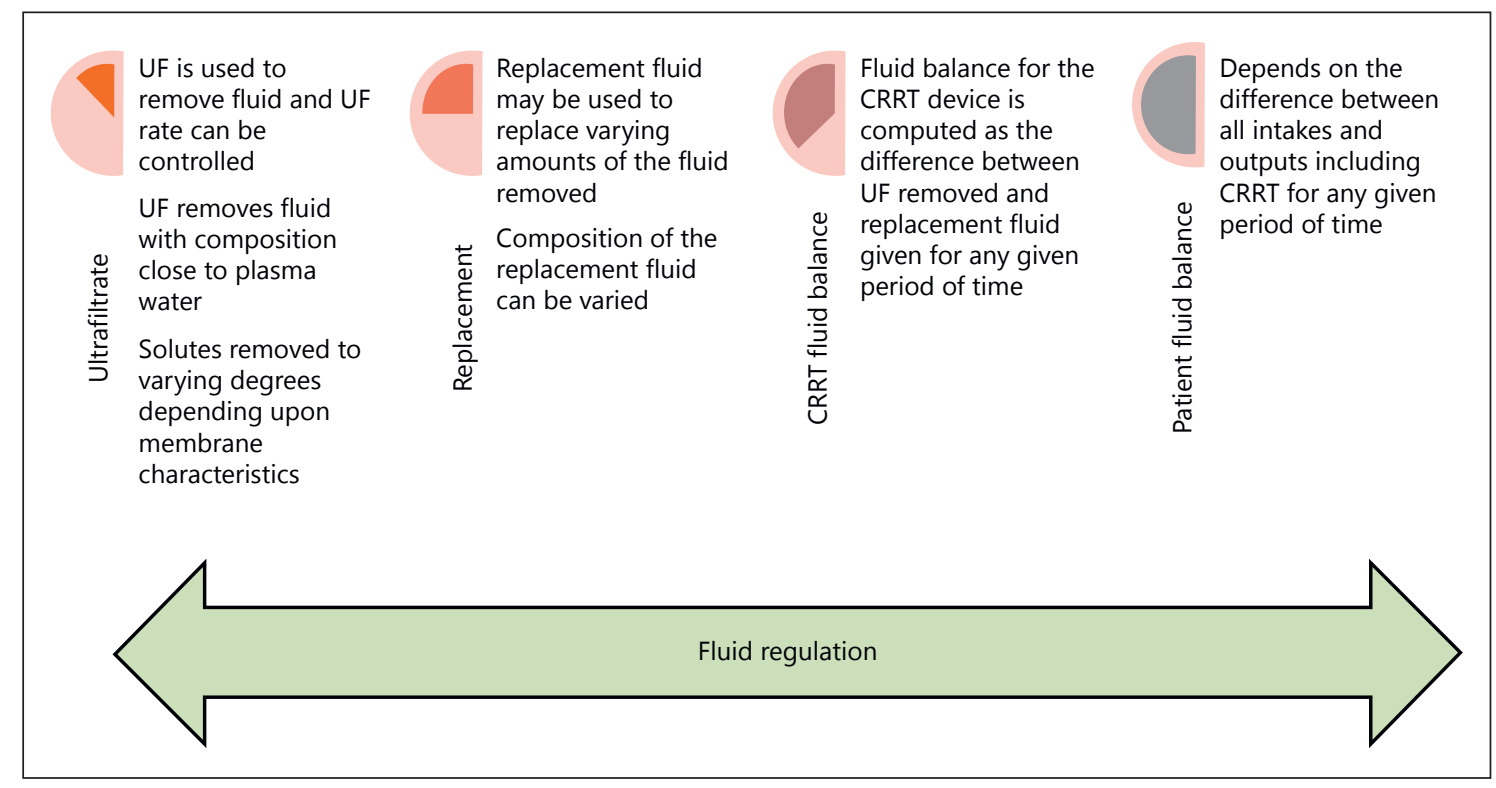

Fig. 2. Principles of fluid management in CRRT. Fluid regulation encompasses all components of fluid management in the patient undergoing CRRT and includes: CRRT machine balance (UF and replacement fluid use) and patient fluid balance. Reprinted with permission from www.ADQI.org.

The decision to move to mechanical fluid removal also entails choosing a modality to achieve this goal such as intermittent hemodialysis/UF, slow low-efficiency dialysis, peritoneal dialysis or CRRT (included in this is SCUF). This decision should be made by taking into account: (1) the total amount of fluid that needs to be removed to achieve clinical goals, (2) ongoing fluid administration needs as well as ongoing net inputs and outputs, (3) hemodynamic status (blood pressure, need for ongoing pressor support), (4) rate at which fluid needs to be removed from the patient, (5) need for solute removal, electrolyte correction or control of uremia and (6) resources available at a particular institution. CRRT affords great flexibility in addressing these issues, as the rate and amount of fluid removal can be continuously and safely adjusted as dictated by patient needs and also allows for institution of dialysis to support other needs of the patient.

\section{What Are the Principles for Fluid Management with CRRT?}

CRRT can be used to achieve fluid regulation in any given patient (fig. 2). In order to achieve fluid regulation, it is critical to understand the differences between machine balance and net patient fluid balance. The balance of anticoagulation, UF and replacement fluid rates will determine the CRRT machine balance. This machine balance can then be added and adjusted to net patient inputs and outputs to influence patient fluid balance (fluid regulation) [20]. Table 5 shows operating characteristics of CRRT and differences between fluid removal and fluid regulation in a given patient. It is important to recognize that precise fluid regulation using CRRT needs to consider not only the dynamic changes in machine fluid balance, but also integrate machine fluid balance with dynamic changes in patient fluid balance. For instance, if the clinician considered just the inputs and outputs in the CRRT but did not take into accounts the various inputs (such as intravenous fluids) and outputs (such as drain outputs) in the patient, then the balance of fluid therapy would be inadequate and the patient could be at risk for either fluid overload or excessive fluid losses. Both the machine and patient fluid balance should be assessed at fixed intervals (e.g., 1-2 h) and has to be combined to estimate the machine-patient integrated fluid balance (iBalance). Figure 3 shows the conceptual model for machine-patient fluid balance at 2 different time points, the concept of iBalance and also the dynamic changes in iBalance.

What Are the Physiological Targets and Goals for Fluid Management with CRRT?

A patients' need for fluid regulation (net iBalance) is determined by the underlying patient characteristics, clinical goals, type of disease, severity of illness and co- 
Fig. 3. Integrated Balance (iBalance). The machine fluid balance (grey circle) depends on UF, replacement fluid rates and anticoagulation. The net patient fluid balance (red circle) is calculated as the algebraic sum of patient inputs (e.g., blood compounds, drugs, nutrition) and outputs (e.g., urine output, drains, insensible losses). The machine-patient integrated fluid balance (iBalance) (blue arrow), which derives from the combination of the machine and the net patient fluid balance, is achievable only when frequent assessment of fluid inputs and outputs and CRRT fluid balance machine parameters are performed (e.g., every 1-2 h). The more frequent the assessment, the shorter is the time gap (vertical axis) and more precise the iBalance (horizontal axis). Reprinted with permission from www.ADQI.org.

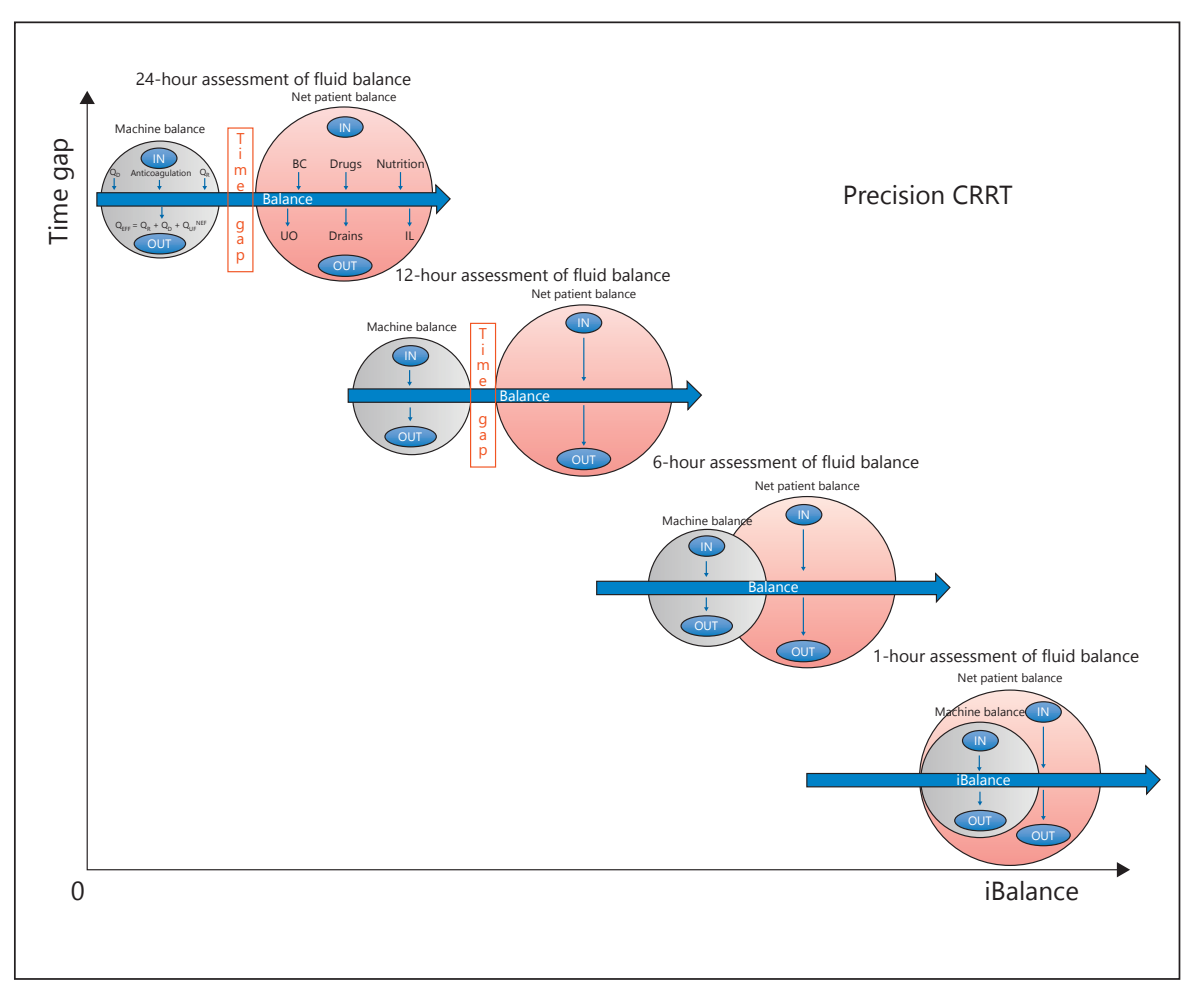

Table 5. Operating characteristics of CRRT - fluid removal versus fluid regulation

\begin{tabular}{lll}
\hline Characteristics & Fluid removal & Fluid regulation \\
\hline UF rate & $\begin{array}{l}\text { To meet anticipated needs based upon static weight at } \\
\text { beginning of treatment as compared to target weight }\end{array}$ & $\begin{array}{l}\text { Variable and reassessed frequently depending upon } \\
\text { patient needs and goals of therapy }\end{array}$ \\
\hline Fluid management & Adjust UF & Adjust amount of replacement fluid and/or UF \\
\hline Fluid balance & Even or negative & Positive, even, or negative \\
\hline Volume removed & Based on physician estimate & Driven by patient characteristics and goals \\
\hline Application & Easy, similar to IHD & Requires specific tools and training \\
\hline IHD = Intermittent hemodialysis. & \\
\hline
\end{tabular}

morbidities. At the start of CRRT, the fluid management strategy should be guided by the patients hemodynamic and volume status. CRRT parameters should be adjusted on a dynamic basis to achieve goals of organ support and should be guided by parameters of patients' end organ dysfunction. It should be stressed that frequent reassessment of these goals is critical and one of the advantages of CRRT is that this single therapy can be tailored to meet rapidly changing clinical needs [21].

Online supplement figure S2 shows the different stages of fluid resuscitation and the conceptual model for flu- id regulation. Typically, fluid removal using CRRT is started during the de-escalation phase of fluid resuscitation [22]. However, in order to achieve clinical goals, CRRT initiation could be considered during the optimization and stabilization phases of resuscitation (for example, a patient who is difficult to ventilate due to massive pulmonary edema may benefit from net fluid removal during earlier stages of resuscitation).

An important principle governing fluid removal during CRRT is the concept of plasma refilling rate. During mechanical fluid removal, fluid is primarily removed 
from the intravascular compartment [23]. Plasma refilling rates from the interstitial compartment will determine the rate of change of the intravascular blood volume. If the UF rate exceeds the plasma refilling rate, decreased blood volume ensues and contributes to hemodynamic instability. Thus, the CRRT prescription for fluid regulation should take into account the overall goal for fluid removal (net removal), the rate of removal (how much fluid is removed in a given time period), patient's volume status and the hemodynamic stability of the patient. Unfortunately, there is no way to easily predict the plasma refilling, and thus, a cautious 'trial and error' approach to fluid removal starting with low fluid removal rates and titrating upward as tolerated is a reasonable approach.

While clinical data that supports one mechanical modality for fluid removal over another are lacking, it is assumed that maintaining hemodynamic stability and organ perfusion during fluid removal is critically important. In this regard, CRRT offers several advantages over intermittent therapies - data from stable outpatients receiving hemodialysis suggest a maximum fluid removal rate of $13 \mathrm{ml} / \mathrm{kg} / \mathrm{h}$ in order to minimize complications such as cardiac stunning or neurological sequelae [24]. Although no studies have specifically addressed this issue, a reasonable assumption would be that patients with AKI and fluid overload also not exceed fluid removal rates of $>13 \mathrm{ml} / \mathrm{kg} / \mathrm{h}$. Thus, for a $70-\mathrm{kg}$ male, the maximum hourly fluid removal rate would be $910 \mathrm{ml} / \mathrm{h}$. Thus, intermittent dialysis or UF would not be appropriate and risks decrease in organ perfusion if the calculated required daily fluid removal rate is greater than $910 \mathrm{ml} / \mathrm{h}$ multiplied by the duration of the session (typically $3-4 \mathrm{~h}$ ). CRRT, on the other hand, by virtue of its slow and continuous fluid removal enables the vascular space to have time to refill from other body fluid compartments and lessens the impact of fluid removal on organ perfusion [25]. In support of this concept is that retrospective studies have suggested that intermittent mechanical therapies as compared with continuous modalities are associated with a high rate of non-recovery from AKI perhaps due to repeated hypotensive (ischemic) events [26].

A unique feature of CRRT is that the correction of water and sodium balance can be separated. UF removes fluid with the same concentration as plasma water and replacement solutions can be administered that are hypotonic (have less sodium) to this removed fluid. This ability allows not only correction of hypernatremia but also removal of excess retained sodium, which allows for cor- rection of extracellular volume overload. Similarly utilizing a hypertonic sodium solution can permit higher sodium levels without affecting the fluid balance.

\section{How Can Fluid Removal and Balance Be Achieved?}

Fluid management is achieved by changing either the amount of UF and/or the replacement fluid. The net amount of UF and replacement fluids may need to be adjusted in frequent intervals. It is important to recognize that if effluent volume is modified, this will affect solute clearance (dose). In order to achieve fluid homeostasis, the CRRT machine fluid balance parameters need to be integrated with the patient's fluid balance, since the CRRT machine balance does not take into account patients' fluid input and output. Figure 4 illustrates 2 different methods of CRRT prescription by integrating the patient and machine fluid balance (iBalance) on an hourly basis to achieve target net negative fluid balance in the patient. In method A, the net UF rate is varied each hour, and in method B, the replacement fluid is varied to achieve a net negative target fluid balance. Hybrid strategies of variable UF and replacement fluid rates can also be used to meet the goals of the patient. Table 6 shows the advantages and disadvantages of the 2 methods.

Statement 9: Clinicians must continuously assess fluid balance and set targets for this variable. These targets should be used to inform decision-making on timing and methods for fluid removal strategies, whether they are pharmacological or mechanical.

\section{Precision CRRT}

\section{How Can the Different Domains of Fluid}

Management Be Integrated to Achieve the Goals of Care?

The patients' goals for fluid management need to be defined initially and adjusted according to the patient's clinical condition. These goals will determine the choice and composition of fluids (dialysate and replacement) and the operational characteristics (e.g., modality, site of fluids delivery and net UF rates) for achieving fluid balance. Fluid overload before CRRT initiation is indeed related to bad outcome. But the prognosis may be improved if the fluid overload is well controlled after CRRT initiation, so the fluid overload after CRRT initiation or during the whole ICU stay may be more important and can be a better predictor of the outcome [27]. The operational characteristics as well as the fluid choices clearly influence one another and it is best to think about the CRRT circuit 
Fig. 4. Approaches to fluid regulation with CRRT fluid balance. Two different methods can be used to achieve the target fluid balance with CRRT. In method A, the net UF rate is varied on an hourly basis. In method $\mathrm{B}$, the net UF rate is fixed and the replacement fluid is varied. Hybrid strategies of variable UF and replacement fluid rates can also be utilized. Reprinted with permission from www.ADQI.org.

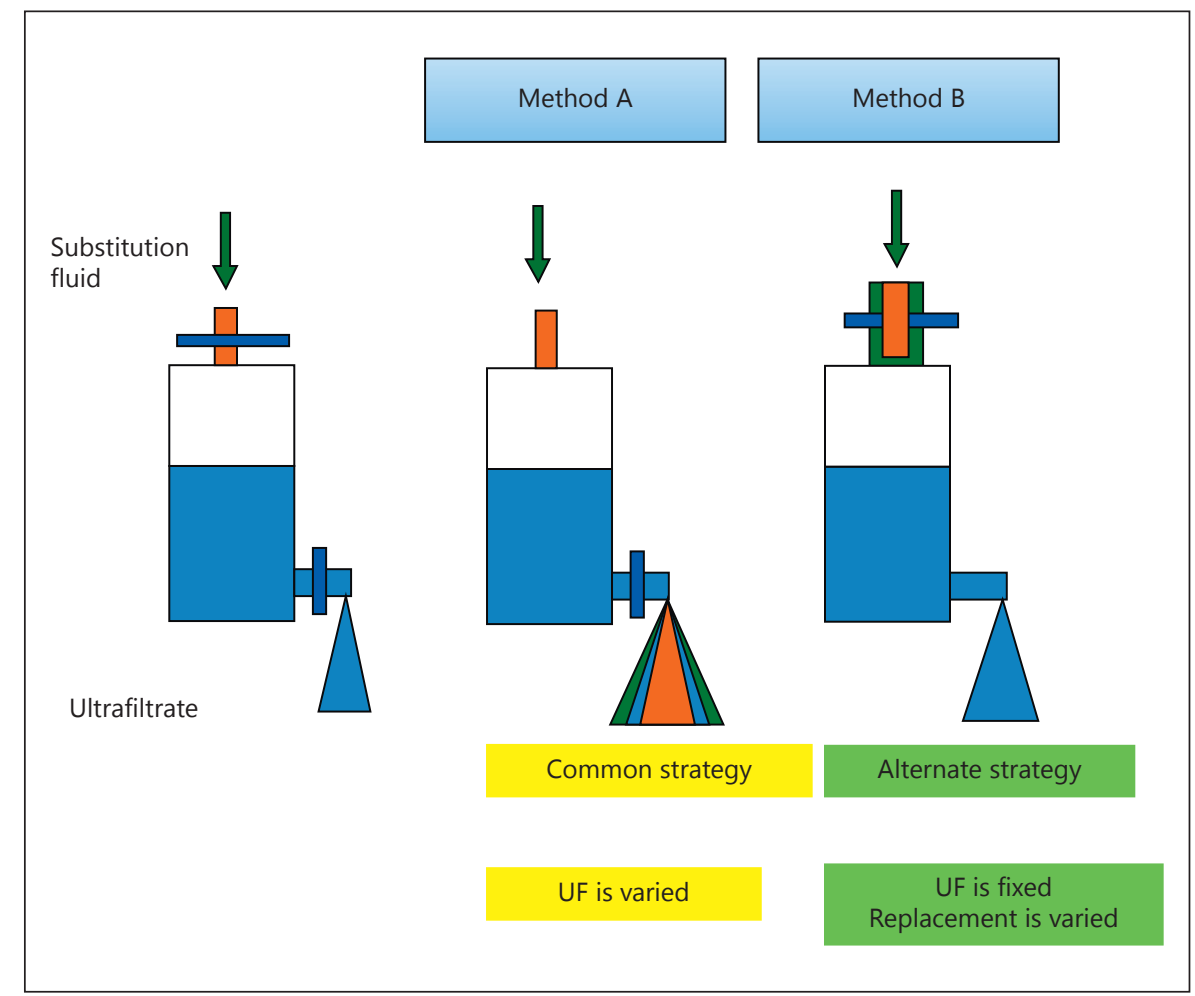

Table 6. Two different methods for fluid balance in CRRT

\begin{tabular}{lll}
\hline Variable & Ultrafiltration technique & Replacement fluid technique \\
\hline Fluid balance & Achieved by varying UF rate & Achieved by adjusting amount of replacement fluids \\
\hline Differences & $\begin{array}{l}\text { Output is varied to accommodate changes in intake } \\
\text { and output to reach a fluid removal goal }\end{array}$ & $\begin{array}{l}\text { Output is fixed to achieve solute clearance goal and } \\
\text { replacement fluid rates are changed to allow flexibility in } \\
\text { reaching net fluid balance goals }\end{array}$ \\
\hline Advantages & $\begin{array}{l}\text { Familiar strategy from intermittent HD } \\
\text { Can allow for fluid balance calculations over an } \\
\text { extended period with calculation of a rate per unit time }\end{array}$ & $\begin{array}{l}\text { Allows for constant solute clearance } \\
\text { Dissociates clearance parameters from fluid balance }\end{array}$ \\
\hline Disadvantages & $\begin{array}{l}\text { Solute clearance may fluctuate } \\
\text { Requires frequent interactions with CRRT machine } \\
\text { to adjust UF rates to meet patient needs }\end{array}$ & $\begin{array}{l}\text { Requires hourly calculations of the amount of replacement } \\
\text { fluid to be given with risk for fluid imbalance if rate not } \\
\text { calculated correctly with clear appreciation of all of the } \\
\text { inputs and outputs for the patient }\end{array}$ \\
\hline
\end{tabular}

as a series of interconnected and interdependent modules. As an example of this interdependence, an increase in UF rate will result in increased $\mathrm{FF}$, requiring a change in $\mathrm{Q}_{\mathrm{B}}$, anticoagulation or pre-filter replacement solutions.

Furthermore, the opportunity to adjust dialysate and replacement solution composition at various sites can be utilized to achieve the desired goals or tailored to spe- cific needs (e.g., hypo/hypernatremia, acid-base balance) [9]. For instance, during CRRT, hypernatremia can be corrected by adding small pre-calculated amounts of a hypertonic sodium chloride solution to the dialysate/replacement fluid bags aiming for a $\left[\mathrm{Na}^{+}\right]$in the fluid that allows safe equilibration and correction of the serum $\left[\mathrm{Na}^{+}\right]$. Conversely, to correct hyponatremia safely, pre-calculated amounts of sterile water can be added 
Fig. 5. Step-wise fluid management prescription in CRRT. Reprinted with permission from www.ADQI.org.
Step 1: Determine the effluent rate (dialysate and/or ultrafiltrate) needed to meet clearance goals (recommend starting at $30 \mathrm{ml} / \mathrm{kg} / \mathrm{h}$ )

- Monitor clearance

- Adjust effluent rate to meet clearance goals

- Monitor hemofilter performance (FUN/BUN)

Step 2: Determine fluid balance needs for the patient and determine the iBalance by incorporating machine and patient fluid balance to determine net goals

- Frequent clinical assessment of fluid removal goal

- Flow sheets to monitor machine/patient balance

- Consider measures of dynamic fluid assessment

Step 3: Determine composition of replacement and/or dialysate solutions to meet goals of maintaining electrolyte and acid-base homeostasis

- Monitor serum electrolytes

- Monitor acid-base status

- Adjust fluids accordingly to meet goals

Step 4: Determine the timing for achievement of goal and monitoring parameters
- Timing based upon hemodynamic stability and imperatives based upon clinical goals

- Set fluid removal rate

- Determine best method to monitor changes in to achieve a fluid $\left[\mathrm{Na}^{+}\right]$that equals the desired target serum $\left[\mathrm{Na}^{+}\right][28,29]$. In either case, correction of the sodium disorder can be achieved through manipulation of the sodium concentration in the dialysate, replacement solution or both and through slow, step-wise changes the desired plasma sodium concentration can be reached.

Statement 10: The goals of care should define the utilization of solutions and fluid balance. Achieving overall fluid goals requires characterization of operating characteristics of CRRT. Flexibility of changing plasma composition and volume status independently could be tailored to the individual need of the patient. It is important to recognize that modifying either circuit integrity or plasma composition or fluid balance will influence the other components.

\section{Stepwise Methodology for Prescription of Fluid Regulation}

What Are the Key Components of a CRRT

Prescription to Achieve Goals of Plasma Composition and Fluid Balance?

Figure 5 shows the key components of a typical CRRT fluid prescription. First, it is important to determine the effluent volume/hour (UF and/or dialysate flow) required to provide adequate solute clearance (we recommend setting this rate at a minimum of $30 \mathrm{ml} / \mathrm{kg} / \mathrm{h}$ to ensure delivery of adequate solute clearance). Second, the volume of net UF needed to achieve fluid balance including a reduction of a given percentage of fluid overload should be estimated. Third, the composition of the fluid needed to replace the fluid removed and to meet the goal for the plasma composition (electrolytes and acidbase) should be determined. Fourth, the timing for achieving the goals and the parameters that need to be monitored should be prescribed. All CRRT prescription strategies should also consider the type of machine, the pump used, the type and frequency of monitoring, charting of data and also the available staffing resources and their experience.

\section{Monitoring}

Monitoring of the circuit integrity, plasma composition, fluid balance and temperature are paramount for optimal delivery of CRRT and to avoid complications. Circuit integrity can be monitored using periodic visual checks of the circuit for any blood clots or air bubbles. Filter efficacy should be monitored using ratio of effluent 
fluid to blood urea nitrogen (FUN/BUN ratio) [30]. Anticoagulation efficacy should be frequently monitored using APTT or anti-Xa levels for heparin or low molecular weight heparin anticoagulation, and post-filter calcium for citrate-based anticoagulation. Access pressures should be monitored to determine adequate circuit blood flow and to troubleshoot any early circuit issues. Plasma composition should be frequently assessed using serum chemistries (e.g., 6-12 h). Hemodynamic parameters, volume status, temperature and body weight should also be monitored (online suppl. table S2). Complications during CRRT can occur due inappropriate goal setting, removing too much or too little volume or due to hemodynamic instability and hypotension.

Given the risks of hypotension and decreases in organ perfusion with fluid removal, close monitoring of the patient's hemodynamic status and tolerance to fluid removal are required. While numerous static and dynamic monitoring techniques are available such as online changes in Hct (relative blood volume), bioimpedance, pulse and stroke volume variation and others, none of these have been sufficiently validated in predicting hypotension in response to fluid removal as well as the adequacy of fluid removal [31]. Thus frequent monitoring of blood pressure and adjustment of fluid removal rates in response to blood pressure changes may be suitable for most patients.

Statement 11: It is recommended that a patient's hemodynamic response to fluid removal be monitored closely and fluid removal rates be adjusted to maintain hemodynamic stability. No study has demonstrated the superiority of one technique of monitoring over others and clinicians should, at a minimum, monitor changes in blood pressure at frequent intervals.

Statement 12: Given the difficulties in predicting how an individual patient may respond to mechanical fluid removal, a reasonable approach is to start with a low rate of fluid removal and slowly increase this to the maximally tolerated rate while monitoring hemodynamic response and avoiding hypotension and decreases in endorgan perfusion. The patient's response to net fluid removal should be reassessed frequently.

Statement 13: Each unit utilizing CRRT should develop a standardized order set to facilitate fluid management orders as well as charting parameters and a standardized flow sheet that ideally can be contained within an electronic medical record. Charting should distinguish CRRT machine parameters from patient fluid balance parameters and integrate these to provide a complete fluid regulation analysis.

Fluid Management in CRRT
Statement 14: Fluid management by CRRT influences patients' core temperature. Core temperature should therefore be monitored continuously.

\section{Research Questions}

Despite several recent advances in technology and delivery of CRRT, a number of questions still remain with regards to fluid prescription.

\section{When Is the Right Time to Initiate CRRT for Fluid/ Volume Management?}

- What data informs this decision?

- Is fluid removal associated with improved outcomes?

\section{What Is Best Practice to Achieve Desired Fluid} Balance with CRRT?

- What is the ideal or target fluid balance that one should strive for in a given patient?

- How should fluid balance be achieved (e.g., fluid removal, fluid addition, fluid regulation)?

- How does patient's volume status and fluid management with RRT influence dose of dialysis?

\section{What Are Key Technological Issues?}

- How does fluid management change with different CRRT systems and role of integrated fluid balancing systems?

- How can we standardize technology for achieving fluid management with CRRT?

- What is the role of electronic medical records in tracking and reporting fluid balance?

\section{Conclusions}

During CRRT, fluids are commonly administered to maintain circuit integrity, regulate plasma composition and maintain fluid balance. CRRT allows the flexibility to tailor the fluids administered or removed to the individual needs of the patient and to achieve intended treatment goals. Precision fluid management during CRRT should integrate both machine and patient fluid balance at frequent intervals to tailor treatment to prevent fluid overload.

\section{Disclosure Statement}

The authors declare that they have no conflicts of interest to declare. 


\section{References}

1 Russell JA, Walley KR, Singer J, Gordon AC, Hébert PC, Cooper DJ, et al: Vasopressin versus norepinephrine infusion in patients with septic shock. N Engl J Med 2008;358:877-887.

2 Rosner MH, Ostermann M, Murugan R, Prowle JR, Ronco C, Kellum JA, et al: Indications and management of mechanical fluid removal in critical illness. Br J Anaesth 2014; 113:764-771.

3 Kellum JA, Mehta RL, Angus DC, Palevsky P, Ronco C; ADQI Workgroup: The first international consensus conference on continuous renal replacement therapy. Kidney Int 2002; 62:1855-1863.

4 Gibney N, Cerda J, Davenport A, Ramirez J, Singbartl K, Leblanc M, et al: Volume management by renal replacement therapy in acute kidney injury. Int J Artif Organs 2008; 31:145-155.

5 Bouchard J, Mehta RL: Volume management in continuous renal replacement therapy. Semin Dial 2009;22:146-150.

6 Bihorac A, Ross EA: Continuous venovenous hemofiltration with citrate-based replacement fluid: efficacy, safety, and impact on nutrition. Am J Kidney Dis 2005;46:908-918.

7 Tolwani A, Wille KM: Advances in continuous renal replacement therapy: citrate anticoagulation update. Blood Purif 2012;34:88-93.

8 Joannidis M, Oudemans-van Straaten HM: Clinical review: patency of the circuit in continuous renal replacement therapy. Crit Care 2007;11:218.

9 Macedo E, Mehta RL: Continuous dialysis therapies: core curriculum 2016. Am J Kidney Dis 2016;pii:S0272-6386(16)30097-X.

10 de Pont AC, Bouman CS, Bakhtiari K, Schaap MC, Nieuwland R, Sturk A, et al: Predilution versus postdilution during continuous venovenous hemofiltration: a comparison of circuit thrombogenesis. ASAIO J 2006;52:416-422.

11 van der Voort PH, Gerritsen RT, Kuiper MA, Egbers PH, Kingma WP, Boerma EC: Filter run time in CVVH: pre- versus post-dilution and nadroparin versus regional heparin-protamine anticoagulation. Blood Purif 2005;23:175-180.
12 Uchino S, Fealy N, Baldwin I, Morimatsu H, Bellomo R: Pre-dilution vs. post-dilution during continuous veno-venous hemofiltration: impact on filter life and azotemic control. Nephron Clin Pract 2003;94:c94-c98.

13 Davenport A: Renal replacement therapy in the patient with acute brain injury. Am J Kidney Dis 2001;37:457-466.

14 Han SS, Bae E, Kim DK, Kim YS, Han JS, Joo KW: Dysnatremia, its correction, and mortality in patients undergoing continuous renal replacement therapy: a prospective observational study. BMC Nephrol 2016;17:2.

15 Kraus MA: Selection of dialysate and replacement fluids and management of electrolyte and acid-base disturbances. Semin Dial 2009; 22:137-140.

16 Morabito S, Pistolesi V, Tritapepe L, Fiaccadori E: Regional citrate anticoagulation for RRTs in critically ill patients with AKI. Clin J Am Soc Nephrol 2014;9:2173-2188.

17 Zhang L, Chen Z, Diao Y, Yang Y, Fu P: Associations of fluid overload with mortality and kidney recovery in patients with acute kidney injury: a systematic review and metaanalysis. J Crit Care 2015;30:860.e7-e13.

18 Malbrain ML, Marik PE, Witters I, Cordemans C, Kirkpatrick AW, Roberts DJ, et al: Fluid overload, de-resuscitation, and outcomes in critically ill or injured patients: a systematic review with suggestions for clinical practice. Anaesthesiol Intensive Ther 2014; 46:361-380.

19 O'Connor ME, Prowle JR: Fluid overload. Crit Care Clin 2015;31:803-821.

20 Davies H, Leslie GD, Morgan D: A retrospective review of fluid balance control in CRRT. Aust Crit Care 2016;pii:S1036-7314(16) 30037-30036.

21 Xu J, Ding X, Fang Y, Shen B, Liu Z, Zou J, et al: New, goal-directed approach to renal replacement therapy improves acute kidney injury treatment after cardiac surgery. J Cardiothorac Surg 2014;9:103.

22 Hoste EA, Maitland K, Brudney CS, Mehta R, Vincent JL, Yates D, et al: Four phases of intravenous fluid therapy: a conceptual model. Br J Anaesth 2014;113:740-747.
23 Daugirdas JT: Pathophysiology of dialysis hypotension: an update. Am J Kidney Dis 2001; 38(4 suppl 4):S11-S17.

24 Flythe JE, Kimmel SE, Brunelli SM: Rapid fluid removal during dialysis is associated with cardiovascular morbidity and mortality. Kidney Int 2011;79:250-257.

25 Rabindranath K, Adams J, Macleod AM, et al: Intermittent versus continuous renal replacement therapy for acute renal failure in adults. Cochrane Database Syst Rev 2007;3: CD003773.

26 Schneider AG, Bellomo R, Bagshaw SM, Glassford NJ, Lo S, Jun M, et al: Choice of renal replacement therapy modality and dialysis dependence after acute kidney injury: a systematic review and meta-analysis. Intensive Care Med 2013;39:987-997.

27 Xu J, Shen B, Fang Y, Liu Z, Zou J, Liu L, et al: Postoperative fluid overload is a useful predictor of the short-term outcome of renal replacement therapy for acute kidney injury after cardiac surgery. Medicine (Baltimore) 2015;94:e1360.

28 Claure-Del Granado R, Bouchard J: Acidbase and electrolyte abnormalities during renal support for acute kidney injury: recognition and management. Blood Purif 2012;34: 186-193.

29 Dangoisse C, Dickie H, Tovey L, Ostermann M: Correction of hyper- and hyponatraemia during continuous renal replacement therapy. Nephron Clin Pract 2014;128:394-398.

30 Sigler MH: Transport characteristics of the slow therapies: implications for achieving adequacy of dialysis in acute renal failure. Adv Ren Replace Ther 1997;4:68-80.

31 Kalantari K, Chang JN, Ronco C, Rosner MH: Assessment of intravascular volume status and volume responsiveness in critically ill patients. Kidney Int 2013;83:1017-1028.

32 Huang Z, Letteri JJ, Ronco C, Gao R, Clark WR: Predilution and postdilution reinfusion techniques; in Ronco C, Bellomo R, Kellum J (eds): Critical Care Nephrology, ed 2. Saunders, 2009, pp 1370-1374. 